\title{
Antibiotic Cement in Arthroplasty: A Meta- analysis of Randomized Controlled Trials
}

Seper Ekhtiari ${ }^{1}$, Thomas Wood ${ }^{1}$, Raman Mundi ${ }^{2}$, Daniel Axelrod ${ }^{1}$, Vickas Khanna ${ }^{3}$, Anthony Adili ${ }^{1}$, Mitchell Winemaker ${ }^{1}$, Mohit Bhandari ${ }^{1}$

1. Orthopaedic Surgery, McMaster University, Hamilton, CAN 2. Surgery, McMaster University, Hamilton, CAN 3. Orthopaedics, McMaster University, Hamilton, CAN

Corresponding author: Seper Ekhtiari, seper.ekhtiari@medportal.ca

\section{Abstract}

\section{Introduction}

Periprosthetic joint infection (PJI) following arthroplasty surgery is a devastating complication. Antibiotic cement has been proposed as a way to reduce PII rates. The aim of this systematic review and meta-analysis was to review all of the available randomized controlled trial (RCT) evidence on the use of antibiotic cement in arthroplasty.

\section{Methods}

PubMed, MEDLINE, and Embase were searched. All records were screened in triplicate. Eligible RCTs were included. Data regarding study characteristics, patient demographics, and rates of superficial and deep infection were collected. The risk of bias was assessed using the Cochrane Risk of Bias Assessment Tool 2.0.

\section{Results}

Five RCTs were included $(n=4,397)$. Four studies compared antibiotic cement to plain cement while one study compared high-dose dual-antibiotic (HDDA) cement to low-dose single-antibiotic (LDSA) cement. The mean age of included patients was 76.4 years (range: 68-83). There was no significant difference in superficial infection rates between antibiotic and plain cement (odds ratio (OR): 1.33, 95\% Confidence Interval $(\mathrm{CI}): 0.77-2.30, \mathrm{p}=0.3)$. There was a large but non-significant reduction in deep infection rates for antibiotic cement (OR: $0.20,95 \% \mathrm{CI}: 0.03-1.32, \mathrm{p}=0.09$ ). There was a significantly lower rate of infection with HDDA as compared to LDSA (OR: $0.31,95 \%$ CI: 0.09-0.88, $\mathrm{p}=0.041$ ).

\section{Conclusion}

The available evidence from RCTs reveals a potential benefit for antibiotic cement in arthroplasty surgery, though this difference is non-significant and highly imprecise. Furthermore, HDDA cement was significantly more effective than LDSA cement. There is a need for large, pragmatic trials on this topic.

Received 03/24/2020

Review began 04/02/2020

Review ended 04/20/2020

Published 04/29/2020

๑) Copyright 2020

Ekhtiari et al. This is an open access article distributed under the terms of the Creative Commons Attribution License CC-BY 4.0., which permits unrestricted use, distribution, and reproduction in any medium, provided the original author and source are credited.
Categories: Infectious Disease, Orthopedics

Keywords: arthroplasty, infection, cement, periprosthetic joint infection, total joint arthroplasty

\section{Introduction}

Across the United States and Canada alone, over one million primary total hip arthroplasty (THA) and total knee arthroplasty (TKA) procedures are performed on an annual basis and the incidence of these procedures is steadily increasing [1-3]. The success of joint replacement surgery in restoring patient function and improving quality of life has been well accepted [4-5]. In fact, THA has been previously deemed the "orthopedic operation of the century" [6].

Complications after hip and knee arthroplasty significantly undermine the success of these elective procedures. Periprosthetic joint infections (PJIs), in particular, remain one of the most devastating complications following hip and knee replacement for physicians and patients alike, as these infections result in increased patient morbidity and mortality, psychological hardship, and a significantly increased financial burden to health care systems [7-9]. Given the heightened concern for PIIs shared universally by surgeons, a variety of peri- and intraoperative practice patterns have emerged to mitigate infection risk. The evidence to inform many of these practices, however, remains equivocal and controversial [10].

The use of antibiotic cement has gained particular attention, despite conflicting evidence for its efficacy in preventing PIIs [11-13]. It remains crucial to clarify the efficacy of bone cement, however, as unnecessary use of antibiotic-laden bone cement (ALBC) leads to needless health care costs and potential concerns with antimicrobial stewardship, though limited data exists on this latter issue specifically. Conversely, if ALBC is truly effective in decreasing infection risk, even modest reductions in infection rates can have a significant 
impact given the number of joint replacements performed annually.

As such, the objective of the current study was to perform a systematic review and meta-analysis of randomized controlled trials (RCTs) evaluating the efficacy of antibiotic bone cement in reducing prosthetic joint infections in patients undergoing primary total hip or knee arthroplasty.

\section{Materials And Methods \\ Protocol}

This systematic review and meta-analysis was conducted in accordance with the Cochrane Handbook for Systematic Reviews of Interventions. The study is presented as per Preferred Reporting Items for Systematic Reviews and Meta-Analyses (PRISMA) [14].

\section{Eligibility criteria}

The focus of this systematic review and meta-analysis was on RCTs assessing the efficacy of antibiotic cement in reducing PJI. Inclusion criteria were as follows: 1) RCT, 2) patients undergoing primary or aseptic revision hip or knee arthroplasty, 3) intervention arm of antibiotic cement, 4) comparator of either plain cement or a different number of antibiotics or dose of antibiotics used in the cement, and 5) available in full text in English through the McMaster University library or available for interlibrary loan from an affiliated university. Aseptic revisions were included given that a limited amount of evidence was expected to be available, and thus a broad overview of this intervention was desired. 'Exclusion criteria were as follows: 1) studies in which revision surgery was being performed for infection, and 2) overlapping reports from the same study cohort; in this case, the study with the longest follow-up while still being powered for the primary outcome, were included.

\section{Information sources}

The search, which was performed on December 19, 2019, included PubMed, MEDLINE, and Embase. All results from database inception to search date were included.

\section{Search}

The search strategy included key terms related to arthroplasty, antibiotic cement, infection, and random allocation. Appendix A outlines the full search strategy.

\section{Study selection}

After completion of the search, duplicates were removed and the studies imported into Rayyan (Qatar Computing Research Institute, Doha, Qatar). Studies were screened in triplicate by independent reviewers. Studies were screened sequentially in two stages: abstract/title screen and full text. Disagreements at the title and abstract stage were handled with automatic inclusion in the next stage. Discrepancies at the fulltext stage were resolved by consensus.

\section{Data collection process}

Data were collected using an online collaborative spreadsheet (Google Sheets, Google LLC, Mountain View, California). The spreadsheet was piloted prior to use and adjustments made as necessary. Each reviewer's data were audited by the other two reviewers for accuracy.

\section{Data items}

Data collected included study characteristics, patient demographics, allocation and randomization techniques, and blinding. In addition, details of the intervention and control arms were collected, along with follow-up timelines. Finally, superficial and deep infection, as well as revision outcomes, were collected.

\section{Risk of bias in individual studies}

Risk of bias among individual studies was assessed using the Cochrane Risk of Bias Assessment Tool 2.0 [15].

\section{Summary measures}

Demographic data is presented using a descriptive statistic, with mean \pm standard deviation (SD) for normally distributed data and median \pm interquartile range (IQR) for non-parametric data. For comparing dichotomous outcomes, an odds ratio (OR) with 95\% Confidence Interval (CI) is presented.

\section{Synthesis of results}

A pairwise meta-analysis was performed to compare intervention and control arms for superficial infection, deep infection, and revision rates. A random-effects (RE) model was employed if there was significant 
heterogeneity, otherwise, a fixed-effects (FE) model was utilized. Heterogeneity was assessed using the chisquared statistic, presented as an I2 value, and considered to be significant if $\mathrm{p}<0.1$. For all other tests, significance was set at the $\mathrm{p}<0.05$ level a priori.

\section{Results}

\section{Study selection}

The search strategy returned 667 results. Ultimately, five RCTs were included. See Figure 1 for the PRISMA flow diagram [14].

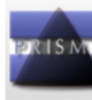

PRISMA Flow Diagram
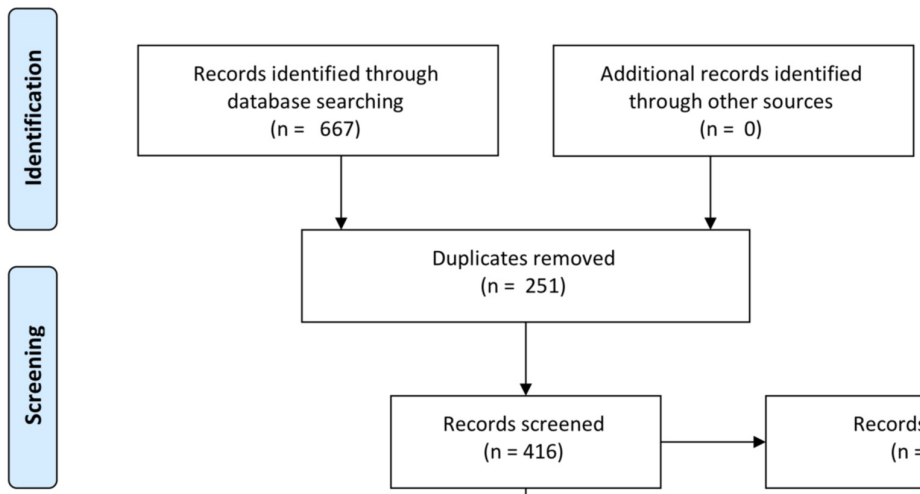

Duplicates removed

$(n=251)$
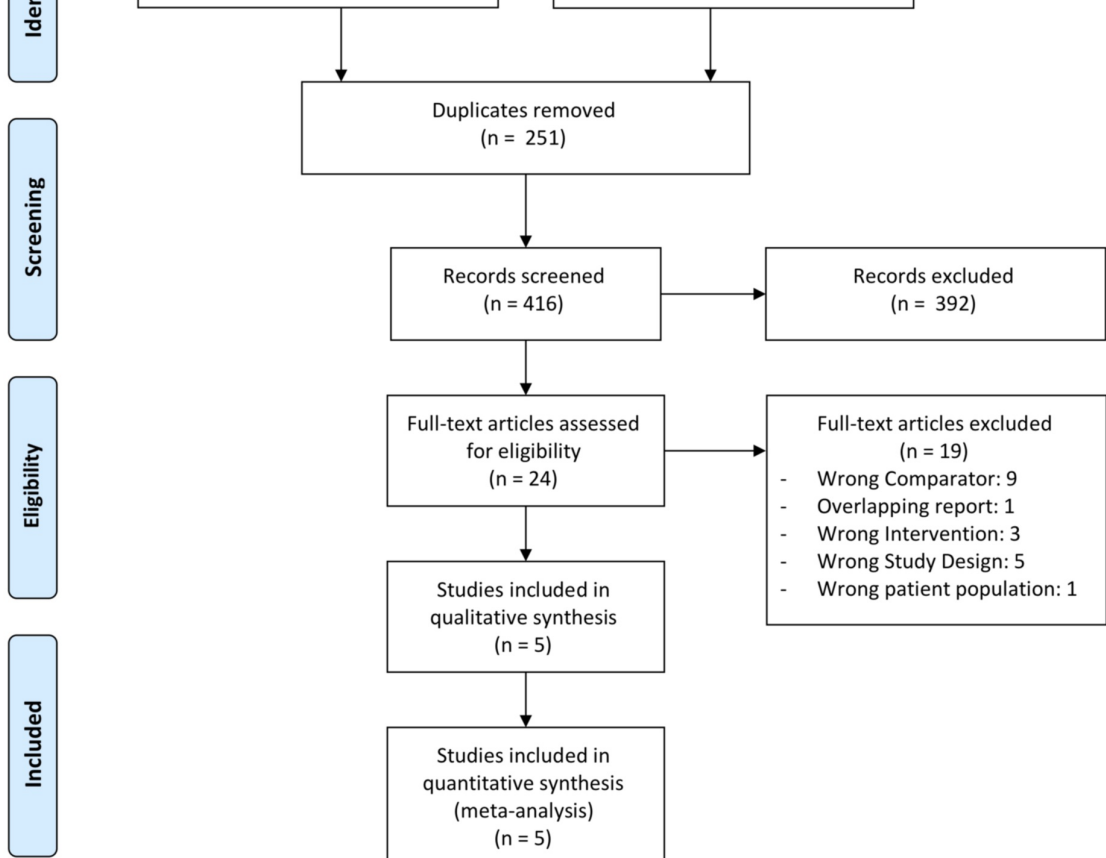

$(n=5)$

tudies included in

quantitative synthesis

(meta-analysis)

$(n=5)$

FIGURE 1: PRISMA flow diagram

\section{Study characteristics}

The five included studies were published between 2001 and 2016 and enrolled a total of 4,397 patients [1620]. Four studies compared antibiotic cement to plain cement while one study compared high-dose dualantibiotic (HDDA) cement to low-dose single-antibiotic (LDSA) cement [16-20]. Overall, 2,643 patients underwent procedures with antibiotic cement while 1,754 underwent procedures with plain cement. Seventy percent of enrolled patients were female $(3,076 / 4,397)$, and the weighted mean age of all included patients was 76.4 years old (range: 68-82.96). Three studies assessed patients undergoing primary TKA $(n=3549)$, one study assessed those undergoing aseptic revision TKA $(n=183)$, and one study assessed patients undergoing hip hemiarthroplasty for fracture $(n=848)$. Cefuroxime was the active antibiotic in one study while vancomycin, gentamicin, erythromycin were used in one study each. One study used a combination of gentamicin and clindamycin in the HDDA group. Table 1 details the characteristics of the included studies. 


\section{Cureus}

\begin{tabular}{|l|lllll|}
\hline Lead Author & Year & Country & Sample Size & Female (\%) & Mean Age (years) \\
\hline Chiu [18] & 2001 & China & 78 & 32.1 & 70.6 \\
Chiu [20] & 2002 & China & 340 & 30.3 & 69.0 \\
Chiu [19] & 2009 & China & 183 & 36.6 & 70.5 \\
Hinarejos [17] & 2013 & Spain & 2948 & 76.3 & 75.9 \\
Sprowson [16] & 2016 & United Kingdom & 848 & 74.5 & 82.6 \\
\hline
\end{tabular}

TABLE 1: Characteristics of included studies

\section{Synthesis of results}

Superficial Infection

The four studies comparing antibiotic cement to plain cement all reported superficial infection rates [17-20]. None of the studies found a significant effect for antibiotic cement. The pooled superficial infection rate was $1.7 \%(30 / 1795)$ in the antibiotic cement group as compared to $1.3 \%(22 / 1754)$ in the plain cement group. The pooled estimate, comparing antibiotic cement to plain cement, revealed an OR of 1.33 (FE, 95\% CI: 0.77$2.30, p=0.3$ ), slightly favoring plain cement though this was not significant. There was no heterogeneity (I2 $=0 \%$ ). Figure 2 displays the forest plot for superficial infection. The study comparing HDDA to LDSA cement found a superficial infection rate of $0.6 \%(2 / 360)$ in the HDDA group as compared to $1.9 \%(7 / 376)$ in the LDSA group (OR: 0.29, 95\% CI: 0.06-1.43, $\mathrm{p}=0.13)$ [16].

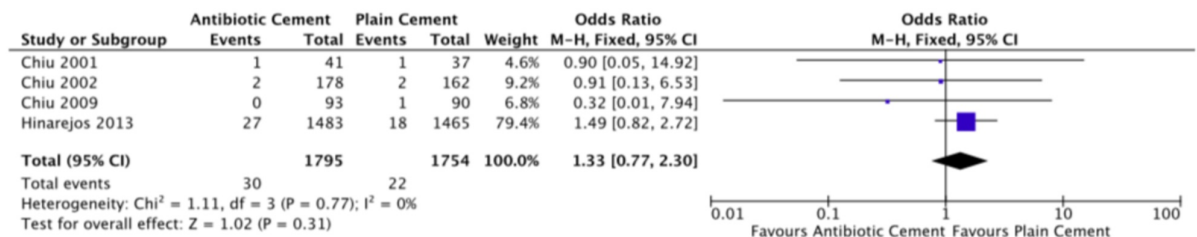

\section{FIGURE 2: Forest plot of superficial infection rates}

Deep Infection

All studies reported deep infection rates. Among the studies comparing antibiotic cement to plain cement, none found a significant effect, though all trended in favor of antibiotic cement [17-20]. Pooled deep infection rate for antibiotic cement was $1.1 \%(20 / 1795)$ as compared to $2.1 \%(36 / 1754)$ for plain cement (OR: 0.20 , RE, 95\%CI: $0.03-1.32, \mathrm{p}=0.09)$. There was moderate heterogeneity $(\mathrm{I} 2=66 \%)$. Figure 3 displays the forest plot for deep infection. The study comparing HDDA to LDSA cement found a significantly lower rate of infection with HDDA (1.1\% vs. 3.5\%, OR: 0.31, 95\% CI: 0.09-0.88, p = 0.041) [16].

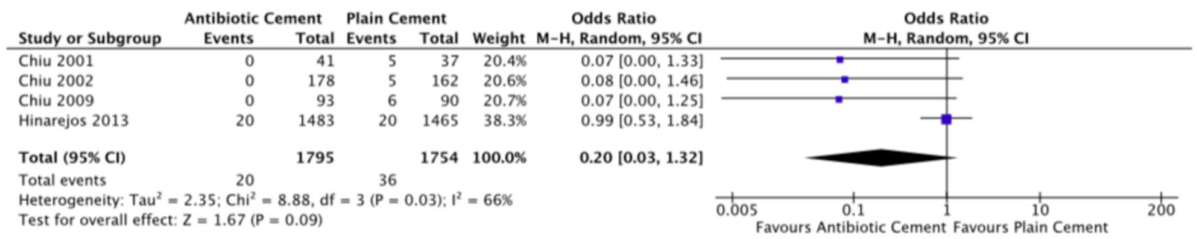

\section{FIGURE 3: Forest plot of deep infection rates}

Risk of Bias Among Included Studies

Of the five included studies, four were found to be at an overall high risk of bias while one study was rated "some concerns" [16-20]. Blinding and random sequence generation were the most domains most frequently at a high risk of bias while all studies had a low risk of bias for missing outcome data. Table 2 details the risk of bias assessment. 


\section{Cureus}

\begin{tabular}{|c|c|c|c|c|c|c|c|}
\hline $\begin{array}{l}\text { Lead } \\
\text { Author }\end{array}$ & $\begin{array}{l}\text { Randomization } \\
\text { Process }\end{array}$ & Blinding & $\begin{array}{l}\text { Intention-to- } \\
\text { treat Analysis }\end{array}$ & $\begin{array}{l}\text { Missing } \\
\text { Outcome } \\
\text { Data }\end{array}$ & $\begin{array}{l}\text { Measurement of } \\
\text { Outcome }\end{array}$ & $\begin{array}{l}\text { Selection of } \\
\text { Reported Result }\end{array}$ & $\begin{array}{l}\text { Overall Risk } \\
\text { of Bias }\end{array}$ \\
\hline $\begin{array}{l}\text { Chiu } 2001 \\
{[18]}\end{array}$ & High risk & High risk & Some concerns & Low risk & Some concerns & Some concerns & High risk \\
\hline $\begin{array}{l}\text { Chiu } 2002 \\
\text { [20] }\end{array}$ & High risk & High risk & Some concerns & Low risk & Some concerns & Some concerns & High risk \\
\hline $\begin{array}{l}\text { Chiu } 2009 \\
\text { [19] }\end{array}$ & High risk & High risk & Some concerns & Low risk & Some concerns & Some concerns & High risk \\
\hline $\begin{array}{l}\text { Hinarejos } \\
2013 \text { [17] }\end{array}$ & Low risk & $\begin{array}{l}\text { Some } \\
\text { concerns }\end{array}$ & High risk & Low risk & Some concerns & Low risk & High risk \\
\hline $\begin{array}{l}\text { Sprowson } \\
2016 \text { [16] }\end{array}$ & $\begin{array}{l}\text { Some } \\
\text { concerns }\end{array}$ & Low risk & Low risk & Low risk & Low risk & Low risk & $\begin{array}{l}\text { Some } \\
\text { concerns }\end{array}$ \\
\hline
\end{tabular}

TABLE 2: Risk of bias across included studies

Additional Analyses

Given that one of the studies included in the quantitative meta-analysis included patients undergoing revision surgery, we performed a post-hoc sensitivity analysis by repeating the meta-analysis without including that study [19]. The results were very similar to the original analysis though with less precision (OR: $0.26,95 \% \mathrm{CI}: 0.03$ to $2.17, \mathrm{p}=0.21$ ). See Appendix B for the forest plot for this sensitivity analysis.

\section{Discussion}

The findings of this systematic review and meta-analysis reveal that despite a total of 4,397 patients having been randomized to receive antibiotic cement or plain cement, the overall effect of antibiotic cement remains unclear. While the pooled effect estimate suggests a very large reduction in rates of PJI, this point estimate is associated with massive confidence intervals that cross the line of no effect. Thus, further highquality evidence is needed in the form of very large, adequately powered, multicenter, and pragmatic RCTs that seek to answer this question definitively.

The presence of deep infection can be caused by either direct infiltration from skin flora and contaminants or hematogenous spread from other sources. In the setting of acute periprosthetic infection, it is assumed that nearly all infections are caused through direct infiltration secondary to the formation of a biofilm [21]. The biofilm reliably forms through four steps: formation of a conditional film, microbial mass transport, anchoring through exopolymer production, and eventual growth of adhering micro-organisms [22]. In-vitro models have demonstrated that antibiotic cement reduces biofilm formation on cement discs after 24 hours [23]. This has been theorized to have occurred secondary to the inhibition of microbial mass transport, as the formation of a conditional film is unaffected by the presence of antibiotic bone cement. Moreover, although the diffusion properties of different antibiotic cement mixtures vary, the general patterns are the same: a large amount of antibiotic is released in the first 24-48 hours, followed by slow diffusion over the next days to weeks postoperatively [22].

Given that antibiotic cement primarily enacts its effects over the course of many weeks, it is logical that it would not prevent infection by inhibiting the creation of the conditional biofilm but rather by reducing bacterial transport to eventual biofilm formation. This is consistent with our finding that antibiotic cement does not reduce the risk of superficial infections, with an infection rate of $1.7 \%$ and $1.3 \%$ in the antibiotic and plain cement groups, respectively. Existing bacteria that colonize the skin during the perioperative period are responsible for the majority of superficial infections [24]. Therefore, antibiotic cement, which works over a period of days to a week, would not likely prevent contamination of the wound with these surface organisms. Rather, perioperative intravenous antibiotic prophylaxis and diligent sterile technique are more likely to reduce superficial surgical site infections [21,25-26].

The dose and type of antibiotic may be an important consideration when selecting an appropriate cement. The studies included in this review utilized five different antibiotics at varying doses, some of which are no longer in common use, which may have contributed to the levels of heterogeneity and imprecision. Furthermore, the rates of elution even among the same antibiotics is different in cement from different manufacturers. Previous literature has discussed the importance of fast-setting cement in the context of arthroplasty [27]. Furthermore, the single study that compared two different doses of antibiotic cement found that the high-dose, dual antibiotic cement was associated with a significantly lower rate of infection 
as compared to a low-dose, single antibiotic cement [16]. Generally, less than $1 \mathrm{~g}$ of antibiotics per $40 \mathrm{~g}$ of cement is considered to be a low dose.

Given the sheer volume of total joint replacements, the findings of this study need to be contextualized within a resource-limited health care system. Costs for antibiotic cement have been reported at nearly $\$ 300$ more per case as compared to plain cement ( $\$ 416$ vs $\$ 117)$ while the total 90 -day costs for a patient requiring revision surgery secondary to PJI are approximately USD 30,000 higher than one without PJI [28-29]. Currently, the annual cost of revision surgeries in Canada is estimated at 163 million Canadian dollars (CAD), and infection is the leading cause of revision surgery, accounting for $31.8 \%$ of all revisions [1]. Thus, even a modest relative risk reduction of $25 \%$ would result in annual savings of nearly 15 million CAD in Canada alone.

The strengths of our review lie in our rigorous methodological approach and the high quality of evidence included. Our review only included the highest quality evidence in randomized controlled trials, we performed title, abstract, and full-text screening in triplicate, and we performed random spot checks on abstraction to ensure accuracy.

This study is limited by the heterogeneity of various interventions such as primary, revision, and hemiarthroplasty. However, all studies showed a relative reduction in deep PJI regardless of the antibiotic used. Furthermore, though the included studies were all RCTs, the number of studies is limited and the pooled estimate is associated with high imprecision.

\section{Conclusions}

Overall, pooled data on the best available evidence demonstrates a high level of imprecision. Thus, there is a need for large, pragmatic trials on this topic in order to provide a definitive answer to this important question. Future trials should consider specific questions, such as the effective antibiotic dose to be used, as well as cost-utility analyses.

\section{Appendices}

\section{Appendix A}

\begin{tabular}{|c|c|c|}
\hline & MEDLINE/Embase & Pubmed \\
\hline Search strategy & $\begin{array}{l}\text { 1. exp Infection/or exp Surgical Wound Infection/ or } \\
\text { infection.mp. } \\
\text { 2. exp Arthroplasty, Replacement, Knee/ or exp Arthroplasty/ } \\
\text { or exp Arthroplasty, Replacement, Hip/ or Arthroplasty.mp. } \\
\text { 3. irrigation.mp. } \\
\text { 4. exp Anti-Infective Agents, Local/ or wound irrigation.mp. or } \\
\text { exp Anti-Bacterial Agents/ } \\
\text { 5. exp Anti-Bacterial Agents/ or antibiotic cement.mp. or exp } \\
\text { Bone Cements/ } \\
\text { 6. } 3 \text { or } 4 \text { or } 5 \\
\text { 7. } 1 \text { and } 2 \text { and } 6 \\
\text { 8. exp Random Allocation/ or random*.mp. } \\
\text { 9. } 7 \text { and } 8\end{array}$ & $\begin{array}{l}\text { ((()(((infection) OR surgical wound infection)) AND } \\
((((() \text { arthroplasty) OR total knee arthroplasty) OR } \\
\text { total knee replacement) OR total hip } \\
\text { arthroplasty) OR total hip replacement) OR joint } \\
\text { replacement)) AND ((((anti-infective) OR anti- } \\
\text { bacterial) OR antibiotic cement) OR bone } \\
\text { cement*))) AND random* }\end{array}$ \\
\hline $\begin{array}{l}\text { Number of papers } \\
\text { retrieved }\end{array}$ & $\begin{array}{l}\text { MEDLINE: } 120 \\
\text { Embase: } 384\end{array}$ & PubMed: 163 \\
\hline
\end{tabular}

FIGURE 4: Search strategy

\section{Appendix B}

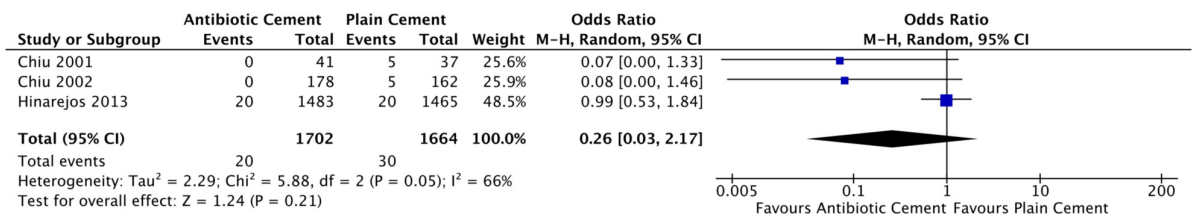

FIGURE 5: Forest plot of the post-hoc sensitivity analysis

\section{Additional Information}

\section{Disclosures}

Human subjects: All authors have confirmed that this study did not involve human participants or tissue. Animal subjects: All authors have confirmed that this study did not involve animal subjects or tissue. Conflicts of interest: In compliance with the ICMJE uniform disclosure form, all authors declare the following: Payment/services info: All authors have declared that no financial support was received from 
any organization for the submitted work. Financial relationships: Seper Ekhtiari declare(s) a grant from St. Joseph's Healthcare Hamilton. Seper Ekhtiari declare(s) a grant from PSI Foundation. Seper Ekhtiari declare(s) a grant from Michael G. DeGroote Fellowship Award. Raman Mundi declare(s) Advisory Board from KCI Medical Canada. Raman Mundi declare(s) Advisory Board from Pendopharm. Raman Mundi declare(s) Speaking fees, Advisory Board from Sanofi. Vickas Khanna declare(s) Consultant from Stryker. Vickas Khanna declare(s) Consultancy from Zimmer Biomet. Anthony Adili declare(s) Consultancy from Sanofi. Mitchell Winemaker declare(s) a grant and Consultancy from Stryker. Mohit Bhandari declare(s) a grant from DJ Orthopaedics. Mohit Bhandari declare(s) a grant from Acumed. Mohit Bhandari declare(s) Consultancy from Ag Novos Healthcare. Mohit Bhandari declare(s) Consultancy from Sanofi Aventis. Mohit Bhandari declare(s) a grant and Consultancy from Stryker . Mohit Bhandari declare(s) Consultancy from Pendopharm. Other relationships: All authors have declared that there are no other relationships or activities that could appear to have influenced the submitted work.

\section{References}

1. Hip and knee replacements in Canada, 2017-2018. (2019). Accessed: Jan 25, 2020: https://secure.cihi.ca/estore/productSeries.htm?pc=PCC51.

2. Etkin CD, Springer BD: The American Joint Replacement Registry-the first 5 years . Arthroplast Today. 2017, 3:67-69. 10.1016/j.artd.2017.02.002

3. Wolford ML, Palso K, Bercovitz A: Hospitalization for total hip replacement among inpatients aged 45 and over: United States, 2000-2010. NCHS Data Brief. 2015, [Epub]:1-8.

4. Geeske Peeters GM, Rainbird S, Lorimer M, Dobson AJ, Mishra GD, Graves SE: Improvements in physical function and pain sustained for up to 10 years after knee or hip arthroplasty irrespective of mental health status before surgery. Acta Orthop. 2017, 88:158-165. 10.1080/17453674.2016.1250059

5. Ethgen $\mathrm{O}$, Bruyère $\mathrm{O}$, Richy $\mathrm{F}$, Dardennes $\mathrm{C}$, Reginster JY: Health-related quality of life in total hip and total knee arthroplasty. A qualitative and systematic review of the literature. J Bone Joint Surg Am. 2004, 86:963974. 10.2106/00004623-200405000-00012

6. Learmonth ID, Young C, Rorabeck C: The operation of the century: total hip replacement . Lancet. 2007, 370:1508-1519. 10.1016/S0140-6736(07)60457-7

7. Gundtoft PH, Pedersen $A B$, Varnum C, Overgaard S: Increased mortality after prosthetic joint infection in primary THA. Clin Orthop Relat Res. 2017, 475:2623-2631. 10.1007/s11999-017-5289-6

8. Mallon CM, Gooberman-Hill R, Moore AJ: Infection after knee replacement: a qualitative study of impact of periprosthetic knee infection. BMC Musculoskelet Disord. 2018, 19:352. 10.1186/s12891-018-2264-7

9. Mallon C, Gooberman-Hill R, Blom A, Whitehouse M, Moore A: Surgeons are deeply affected when patients are diagnosed with prosthetic joint infection. PLoS One. 2018, 13:e0207260. 10.1371/journal.pone.0207260

10. Hatz D, Anoushiravani AA, Chambers MC, El-Othmani MM, Saleh KJ: Approach to decrease infection following total joint arthroplasty. Orthop Clin North Am. 2016, 47:661-671. 10.1016/j.ocl.2016.05.007

11. Schiavone Panni A, Corona K, Giulianelli M, Mazzitelli G, Del Regno C, Vasso M: Antibiotic-loaded bone cement reduces risk of infections in primary total knee arthroplasty? A systematic review. Knee Surg Sports Traumatol Arthrosc. 2016, 24:3168-3174. 10.1007/s00167-016-4301-0

12. Gandhi RD, Backstein D, Zywiel MG: Antibiotic-laden bone cement in primary and revision hip and knee arthroplasty. J Am Acad Orthop Surg. 2018, 26:727-734. 10.5435/JAAOS-D-17-00305

13. Jameson SS, Asaad A, Diament M, et al.: Antibiotic-loaded bone cement is associated with a lower risk of revision following primary cemented total knee arthroplasty: an analysis of 731,214 cases using National Joint Registry data. Bone Joint J. 2019, 101-B:1331-1347. 10.1302/0301-620X.101B11.BJJ-2019-0196.R1

14. Moher D, Liberati A, Tetzlaff J, Altman DG, PRISMA Group: Preferred reporting items for systematic reviews and meta-analyses: the PRISMA statement. PLoS Med. 2009, 6:e1000097. 10.1371/journal.pmed.1000097

15. Tool to assess risk of bias in randomized controlled trials . (2017). Accessed: Feb 2, 2020: https://www.evidencepartners.com/wp-content/uploads/2017/09/Tool-to-Assess-Risk-of-Bias-inRandomized-Controlled-Tria....

16. Sprowson AP, Jensen C, Chambers S, et al.: The use of high-dose dual-impregnated antibiotic-laden cement with hemiarthroplasty for the treatment of a fracture of the hip. The Fractured Hip Infection trial. Bone Joint J. 2016, 98-B:1534-1541. 10.1302/0301-620X.98B11.34693

17. Hinarejos P, Guirro P, Leal J, et al.: The use of erythromycin and colistin-loaded cement in total knee arthroplasty does not reduce the incidence of infection. A prospective randomized study in 3000 knees. J Bone Joint Surg Am. 2013, 95:769-774. 10.2106/JBJS.L.00901

18. Chiu FY, Lin CF, Chen CM, Lo WH, Chaung TY: Cefuroxime-impregnated cement at primary total knee arthroplasty in diabetes mellitus. A prospective, randomised study. J Bone Joint Surg Br. 2001, 83:691-695. 10.1302/0301-620x.83b5.11737

19. Chiu FY, Lin CF: Antibiotic-impregnated cement in revision total knee arthroplasty. A prospective cohort study of one hundred and eighty-three knees. J Bone Joint Surg Am. 2009, 91:628-633. 10.2106/JBJS.G.01570

20. Chiu FY, Chen CM, Lin CF, Lo WH: Cefuroxime-impregnated cement in primary total knee arthroplasty: a prospective, randomized study of three hundred and forty knees. J Bone Joint Surg Am. 2002, 84:759-762.

21. Prokuski L: Prophylactic antibiotics in orthopaedic surgery. J Am Acad Orthop Surg. 2008, 16:283-293. 10.5435/00124635-200805000-00007

22. Gasparini G, De Gori M, Calonego G, Della Bora T, Caroleo B, Galasso O: Drug elution from high-dose antibiotic-loaded acrylic cement: a comparative, in vitro study. Orthopedics. 2014, 37:999-1005. 10.3928/01477447-20141023-57

23. van Loosdrecht MC, Lyklema J, Norde W, Zehnder AJ: Influence of interfaces on microbial activity. Microbiol Rev. 1990, 54:75-87.

24. Ribeiro M, Monteiro FJ, Ferraz MP: Infection of orthopedic implants with emphasis on bacterial adhesion process and techniques used in studying bacterial-material interactions. Biomatter. 2012, 4:176-194. 


\section{Cureus}

10.4161/biom.22905

25. Bratzler DW, Houck PM, Surgical Infection Prevention Guideline Writers Workgroup: Antimicrobial prophylaxis for surgery: an advisory statement from the National Surgical Infection Prevention Project. Am J Surg. 2005, 189:395-404. 10.1016/j.amjsurg.2005.01.015

26. Tyllianakis ME, Karageorgos ACh, Marangos MN, Saridis AG, Lambiris EE: Antibiotic prophylaxis in primary hip and knee arthroplasty. Comparison between cefuroxime and two specific antistaphylococcal agents. J Arthroplasty. 2010, 25:1078-1082. 10.1016/j.arth.2010.01.105

27. Caraan NA, Windhager R, Webb J, Zentgraf N, Kuehn KD: Role of fast-setting cements in arthroplasty: a comparative analysis of characteristics. World J Orthop. 2017, 8:881-890. 10.5312/wjo.v8.i12.881

28. Yayac M, Stein J, Deirmengian GK, Parvizi J, Courtney PM: Conversion total knee arthroplasty needs its own diagnosis-related group code. J Arthroplasty. 2019, 34:2308-2312. 10.1016/j.arth.2019.05.045

29. Kurtz SM, Lau E, Watson H, Schmier JK, Parvizi J: Economic burden of periprosthetic joint infection in the United States. J Arthroplasty. 2012, 27:61-65. 10.1016/j.arth.2012.02.022 